\title{
Net-baryon number fluctuations at finite density
}

\author{
Michał Marczenko* \\ Institute of Theoretical Physics, University of Wroctaw, Plac Maksa Borna 9, PL-50204 \\ Wroctaw, Poland \\ E-mail: michal.marczenko@ift.uni.wroc.pl
}

\section{Krzysztof Redlich}

Institute of Theoretical Physics, University of Wrocław, Plac Maksa Borna 9, PL-50204

Wroctaw, Poland

\section{Chihiro Sasaki}

Institute of Theoretical Physics, University of Wroctaw, Plac Maksa Borna 9, PL-50204

Wroctaw, Poland

\section{Niels-Uwe Friedrich Bastian}

Institute of Theoretical Physics, University of Wroctaw, Plac Maksa Borna 9, PL-50204

Wroctaw, Poland

\begin{abstract}
One of the most significant aspects of QCD thermodynamics is understanding how the transition from hadrons to their constituents—quarks and gluons-relates to the underlying deconfinement and chiral dynamics. This is of major relevance for heavy-ion collisions, as well as in the study of cold and dense systems, such as compact stars. The latter, however, is often studied exclusively in models of either hadron or quark degrees of freedom. In this contribution, we present the mean-field thermodynamics of an effective hybrid quark-meson-nucleon (HQMN) model for QCD phase transitions at low temperatures and finite baryon densities. In this framework, the chiral dynamics is described within the linear sigma model, whereas the deconfinement transition is driven by a medium-dependent modification of the particle distribution functions, where an additional scalar field is introduced. The structure of the net-baryon number fluctuations along with its higher order cumulants is discussed as possible probes for the chiral and deconfinement phase transitions. A qualitative comparison of the results obtained in the nucleonic (parity doublet) and quark (Nambu-Jona-Lasinio) models is also discussed.
\end{abstract}

Critical Point and Onset of Deconfinement - CPOD2017

7-11 August, 2017

The Wang Center, Stony Brook University, Stony Brook, NY

${ }^{*}$ Speaker. 


\section{Introduction}

Recent lattice QCD (LQCD) findings [1,2] suggest the parity doubling structure in the baryonic sector around the chiral crossover temperature. It is found that the masses of the groundstates with positive parity are rather independent of temperature, while the masses of those with negative parity drop significantly towards the transition point, and the two states become degenerate in the vicinity of the transition. This might suggest the chiral symmetry restoration in the baryonic sector of QCD, and should occur also in cold dense matter. This phenomenon can be realized in a schematic framework with chiral symmetry, the parity doublet model [3, 4, 5]. It has been already applied in many aspects of the QCD phenomenology, as well as astrophysical studies of neutron stars $[6,7,8,9,10,11,12,13,14]$.

In this contribution, we consider an extension of the parity doublet model for cold and dense strongly interacting matter, namely the Hybrid Quark-Meson-Nucleon (HQMN) model $[13,14]$. Along with hadrons, the model includes quark degrees of freedom in a standard linear sigma framework. To prevent the unphysical quark population at low density, the HQMN model considers an auxiliary scalar field to which the fermions are coupled to. This field serves as a momentum cutoff in the Fermi-Dirac distribution function, in a way that quarks are suppressed at low density, and likewise, the hadron degrees of freedom at high density.

We study the bulk equation of state and the phase diagram of the HQMN model. We also put our focus on the the behavior of the second- and higher-order cumulants of the net-baryon number density up to the fourth order in the vicinity of the chiral and deconfinement transitions, and discuss their critical behavior.

This paper is organized as follows. In Sec. 2, we introduce the HQMN model setup in the mean-field approximation. In Sec. 3, we discuss obtained numerical results on the equation of state, as well as model phase diagram. In Sec. 4, the structure of the net-baryon number density cumulants is discussed. Finally, Sec. 5 is devoted to the summary and conclusions.

\section{Hybrid Quark-Meson-Nucleon model}

In this section, we introduce the Hybrid Quark-Meson-Nucleon (HQMN) model for the QCD phase transitions at finite temperature and density, following Refs. [13, 14]. Throughout this contribution we consider an isospin-symmetric system of two flavors, $N_{f}=2$, in the mean-field approximation. Instead of the conventional Gel-Mann-Levy model, where the mass of the nucleon is generated through the non-vanishing sigma expectation value, the model considers another realization, where an introduction of a finite mass term does not break the chiral symmetry $[3,4,5]$. Hence, the sigma condensation generates only the mass difference between two chiral partners. The quark sector, on the other hand, is modeled in the standard linear-sigma model.

The thermodynamic potential of the HQMN model in the mean-field approximation is given by the following

$$
\Omega=V_{\sigma}+V_{\omega}+V_{b}+\sum_{x= \pm, q} \Omega_{x}
$$

with

$$
\Omega_{x}=\gamma_{x} \int \frac{\mathrm{d}^{3} p}{(2 \pi)^{3}} T\left[\ln \left(1-n_{x}\right)+\ln \left(1-\bar{n}_{x}\right)\right]
$$




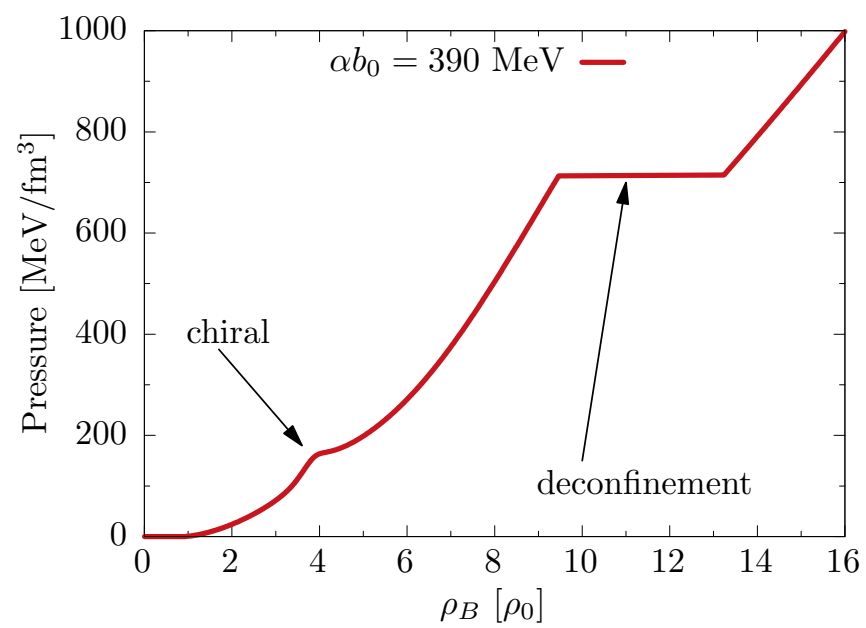

Figure 1: (Color online) Thermodynamic pressure in the HQMN model as function of the net-baryon density for $\alpha b_{0}=390 \mathrm{MeV}$ at temperature $T=10 \mathrm{MeV}$.

where the subscripts \pm denote positive and negative parity nucleons, and $q$ denotes quark. The degeneracy factors $\gamma_{ \pm}=4$ denote the spin-flavor degeneracy for nucleons, $\gamma_{q}=12$ denotes the spin-color-flavor degeneracy factor for quarks. The mean-field potentials read

$$
\begin{aligned}
V_{\sigma} & =-\frac{\lambda_{2}}{2}\left(\sigma^{2}+\pi^{2}\right)+\frac{\lambda_{4}}{4}\left(\sigma^{2}+\pi^{2}\right)^{2}-\varepsilon \sigma, \\
V_{\omega} & =-\frac{1}{2} m_{\omega}^{2} \omega_{\mu} \omega^{\mu}, \\
V_{b} & =-\frac{1}{2} \kappa_{b}^{2} b^{2}+\frac{1}{4} \lambda_{b} b^{4} .
\end{aligned}
$$

The parameters $\lambda_{2}, \lambda_{4}$ and $\varepsilon$ can be connected to the vacuum meson masses and the pion decay constant as

$$
\lambda_{2}=\frac{m_{\sigma}^{2}-3 m_{\pi}^{2}}{2}, \quad \lambda_{4}=\frac{m_{\sigma}^{2}-m_{\pi}^{2}}{2 f_{\pi}^{2}}, \quad \varepsilon=m_{\pi}^{2} f_{\pi},
$$

where the pion mass $m_{\pi}=138 \mathrm{MeV}$, the pion decay constant $f_{\pi}=93 \mathrm{MeV}$. The mass of the sigma meson, $m_{\sigma}$, is treated as a free parameter. In the mean-field approximation, rotational invariance requires that the spatial component of the $\omega_{\mu}$ field vanishes, namely $\langle\omega\rangle=0^{1}$, while $\langle\pi\rangle=0$ follows from parity conservation.

One particular feature of the HQMN model is that it realizes the concept of statistical confinement through a medium-dependent modification of the Fermi-Dirac distribution functions for particles and antiparticles, where an auxiliary scalar field $b$ is introduced, namely

$$
\begin{array}{ll}
n_{ \pm}=\theta\left(\alpha^{2} b^{2}-p^{2}\right) f_{ \pm}, & n_{q}=\theta\left(p^{2}-b^{2}\right) f_{q}, \\
\bar{n}_{ \pm}=\theta\left(\alpha^{2} b^{2}-p^{2}\right) \bar{f}_{ \pm}, & \bar{n}_{q}=\theta\left(p^{2}-b^{2}\right) \bar{f}_{q},
\end{array}
$$

\footnotetext{
${ }^{1}$ In this contribution we denote $\omega_{0} \equiv \omega$.
} 


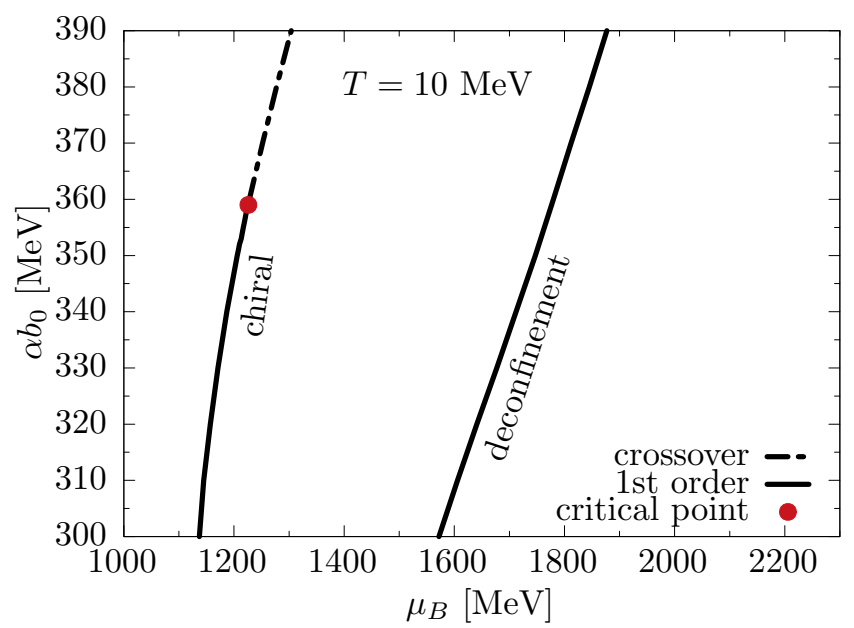

Figure 2: (Color online) The phase diagram of the HQMN model in the $\left(\alpha, \mu_{B}\right)$-plane at temperature $T=$ $10 \mathrm{MeV}$. The first-order transition lines are indicated as black solid lines, while the chiral crossover line is shown as black dash-dotted line. The red circle in the chiral transition line correspond to the critical point.

where $\alpha$ is a dimensionless model parameter. The functions $f_{ \pm}$and $f_{q}$ are standard Fermi-Dirac distribution functions, defined as

$$
f_{x}=\frac{1}{1+e^{\beta\left(E_{x}-\mu_{x}\right)}}, \quad \bar{f}_{x}=\frac{1}{1+e^{\beta\left(E_{x}+\mu_{x}\right)}},
$$

where, for the nucleons, $E_{ \pm}=\sqrt{m_{ \pm}^{2}+p^{2}}$ and $\mu_{ \pm}=\mu_{B}-g_{\omega} \omega$, and for quarks, the dispersion relation is $E_{q}=\sqrt{p^{2}+m_{q}^{2}}$, and the quark chemical potential $\mu_{q}=\frac{1}{3} \mu_{B}$. The $b$ field is generated through the potential $V_{b}$ (see Eq. (2.3)). The expectation value of the field serves as an infrared momentum cutoff for quarks and ultraviolet for nucleons. The potential $V_{b}$ develops a non-trivial vacuum expectation value, and is chosen in a way that it develops non-monotonic behavior that triggers the suppression of quarks at low densities, and nucleon suppression at high densities. The additional $\alpha$ parameter in Eq. (2.5) is a model parameter, whose role is to be studied in the following sections.

The masses of the parity doublers are given by the following relation

$$
m_{ \pm}=\frac{1}{2}\left[\sqrt{\left(g_{1}+g_{2}\right)^{2} \sigma^{2}+4 m_{0}^{2}} \mp\left(g_{1}-g_{2}\right) \sigma\right],
$$

where the couplings $g_{1}, g_{2}$ are the chiral couplings of the fermionic fields in the original parity doublet Lagrangian $[6,13,14]$. The mass of quark is given by

$$
m_{q}=g_{\sigma} \sigma
$$

From Eq. (2.7) and (2.8), it is clear that the chiral symmetry breaking generates only the splitting between the masses of nucleons. When the symmetry is restored, the masses become degenerate according to $m_{ \pm}(\sigma=0)=m_{0}$. Quark mass, on the other hand, is completely generated through the condensation of the $\sigma$ meson. 


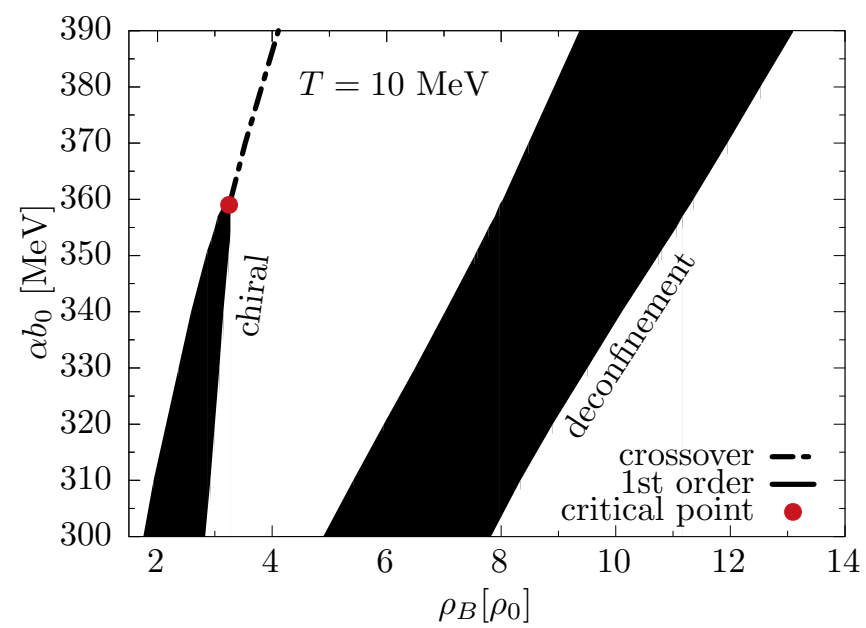

Figure 3: (Color online) The phase diagram of the HQMN model in the $\left(\alpha, \rho_{B}\right)$-plane at temperature $T=$ $10 \mathrm{MeV}$. The first-order transition are depicted as black areas, and correspond to the induced jump in density. The chiral crossover line is shown as black dash-dotted line. The red circle in the chiral transition line correspond to the critical point.

We identify the hadronic states with the nucleon $N(938)$ (positive-parity state) and its resonance $N(1535)$ (negative-parity state). Their vacuum masses are set to be $m_{+}=938 \mathrm{MeV}$ and $m_{-}=1500 \mathrm{MeV}$. Following previous studies $[6,7,11,13]$, we choose the chirally invariant mass to be $m_{0}=790 \mathrm{MeV}$. The model parameters used in this contribution are fixed as in Ref. [14]. The thermal values of the mean fields are determined by extremizing the thermodynamic potential with respect to the mean-fields.

\section{Equation of state}

In this contribution we consider a particular choice of the parameter $\alpha$, namely $\alpha b_{0}=390 \mathrm{MeV}$, where $b_{0}$ is the vacuum expectation value of the $b$ field, and study the thermodynamic quantities at finite temperature $T=10 \mathrm{MeV}$. In this section we focus on the bulk equation of state and the phase diagram of the HQMN model.

In Fig. 1, we show the resulting thermodynamic pressure plotted against the net-baryon density $\rho_{B}$ in the units of nuclear saturation density $\rho_{0}$. The softening of the equation of state around $\rho_{B}=4 \rho_{0}$ is due to a smooth chiral crossover phase transition. The transition point is extracted from the peak of $\partial \sigma / \partial \mu_{B}$ and is located at $\mu_{B}=1305 \mathrm{MeV}$. At higher densities the system undergoes a first-order deconfinement transition, which is triggered by the non-monotonic behavior of the $b$-field expectation value. The transition happens at $\mu_{B}=1868 \mathrm{MeV}$. The jump in density is of the order of $4 \rho_{0}$. From the figure it is evident that the two transitions are separated. Hence, the model predicts a phase in which quarks are still confined but the chiral symmetry is restored.

In Fig. 2, we show the model phase diagram in the $\left(\alpha, \mu_{B}\right)$-plane at $T=10 \mathrm{MeV}$. From the figure it is evident that the chiral and deconfinement transitions are always separated. For higher values of the parameter $\alpha$, the chiral phase transition is a smooth crossover shown as the dashdotted line. At lower values of $\alpha$ it develops a critical point (red circle), and eventually turns into 


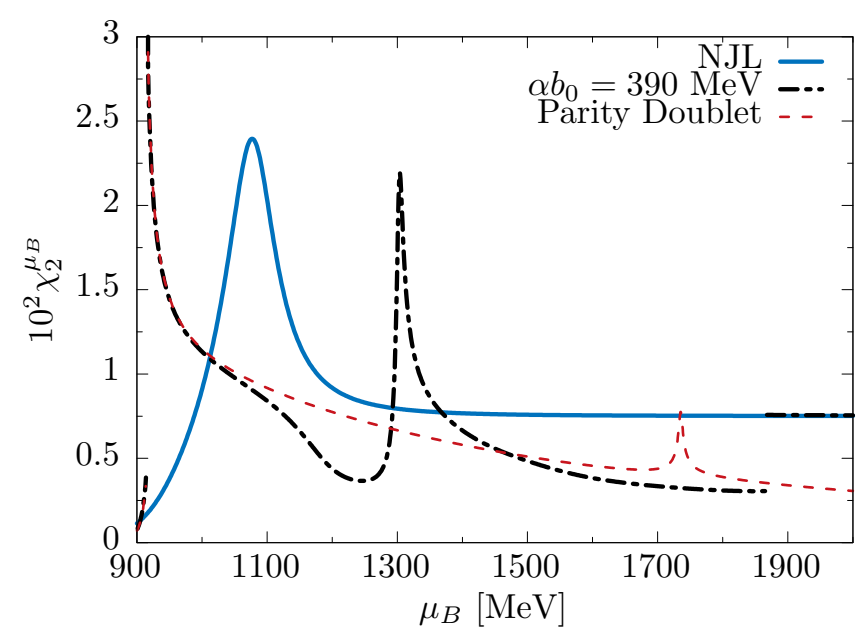

Figure 4: (Color online) Second-order cumulant of the net-baryon number density at temperature $T=$ $10 \mathrm{MeV}$. Shown are results obtained in three models, NJL (blue solid line), parity doublet (red dashed line) and HQMN model for $\alpha b_{0}=390 \mathrm{MeV}$ (black dash-dotted line). Parametrization for the NJL and parity doublet model is from [14]

a 1st-order transition indicated as a solid line. The deconfinement transition is always of 1 st order, irrespectively of the choice of the parameter $\alpha$.

In Fig. 3, we show the phase diagram as a function of the net-baryon number density. We observe that the two transitions are always separated by roughly $3-5 \rho_{0}$. At low values of $\alpha$, the first-order chiral phase transition causes a finite density jump of roughly $2 \rho_{0}$. The strength of the transition shrinks towards the critical point and becomes smooth above it. The jump in density connected with the deconfinement transition is on the other hand roughly of the same order for all choices of $\alpha$, namely $3-4 \rho_{0}$. We note that the phase structure obtained at different temperatures is qualitatively similar to the one studies here [14]. We also remark that in principle, it is possible to modify the phase structure, so that the two transitions happen simultaneously. The transitions would be then of the first order, given by the finite jump in $b$-field expectation value at low temperature [14].

In the next section we study the behavior of the higher-order cumulants of the net-baryon number density.

\section{Net-baryon number density cumulants}

The fluctuations of conserved charges are important quantities because they reveal more information about the matter composition, and are used as probes for the phase transitions. In principle, the critical behavior of chiral models should be universal and follow that of QCD. Namely, at high temperatures, the chiral transition belongs to the $O(4)$ universality class, which at some value of baryon chemical potential is followed by the critical point, which turns into a first-order transition. This is naturally encoded in chiral models, as well as in quark models. Here, we put our focus on the influence of the statistical confinement on the behavior of the higher-order cumulants in the vicinity of phase transitions. 

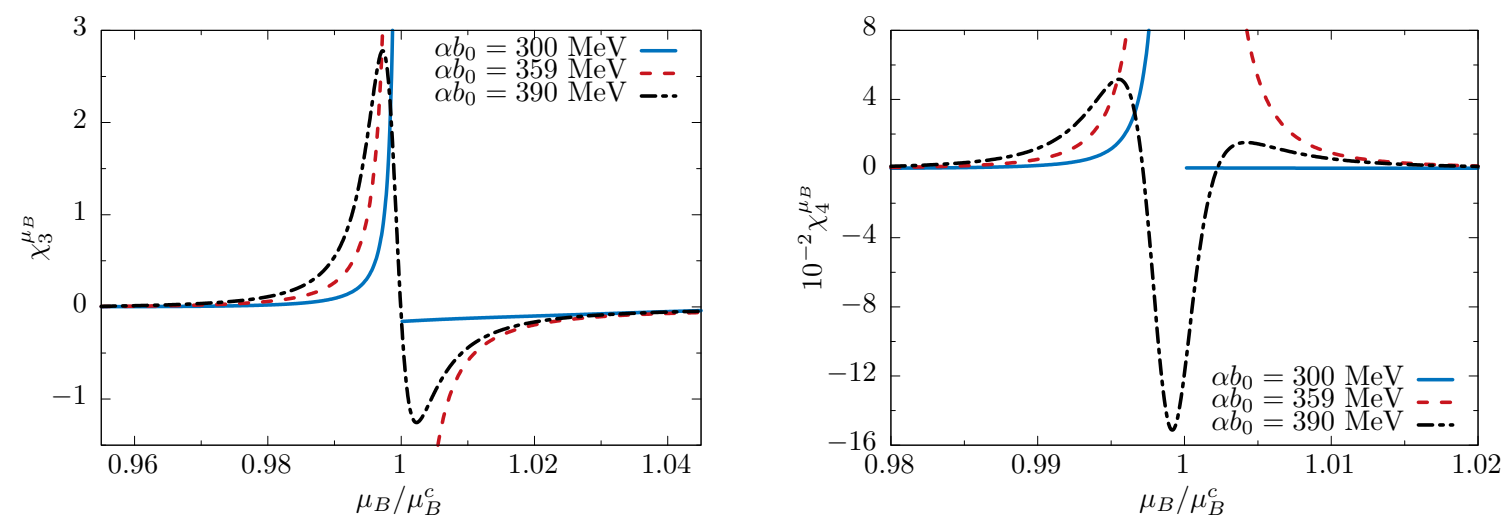

Figure 5: (Color online) Third- (left panel) and fourth-order (right panel) cumulants of the net-baryon number density at $T=10 \mathrm{MeV}$. The blue solid lines correspond the the first-order transition, red dashed line to the second-order transition, and the black dash-dotted line to the crossover transition. The $x$-axis is normalized by the chiral transition value $\mu_{B}^{c}$ in each scenario.

In the grand canonical ensemble, the cumulants are realized as higher-order derivatives with respect to an external parameter. The cumulants net-baryon number are defined as

$$
\chi_{n}=-\frac{\partial^{n} \Omega}{\partial \mu_{B}^{n}}
$$

Unlike in the lattice QCD studies, for the propose of this study, it is more convenient to consider cumulants normalized by the baryon chemical potential [14], namely

$$
\chi_{n}^{\mu_{B}}=\mu_{B}^{n-4} \chi_{n} .
$$

We compare the results obtained in the HQMN model, with the results obtained in the parity doublet model, as well those in the Nambu-Jona-Lasinio (NJL) model. The parity doublet model is a model with only hadronic degrees of freedom, while the NJL consists solely of quarks. The parametrization used here can be found in Ref. [14].

In Fig. 4 we show the second-order cumulants, normalized by the baryon chemical potential, in all three models. The result obtained in the NJL model (blue solid line), undergoes a chiral crossover. This is seen as a peak in the second-order cumulant. At higher baryon chemical potential it approaches the Stefan Boltzmann limit. The parity doublet and HQMN results, develop a jump around $\mu_{B}=917 \mathrm{MeV}$. This is so because both models are fixed to reproduce the nuclear groundstate properties at $T=0$. The parity doublet model develops the chiral peak at higher values of the baryon chemical potential and continuously go to zero in the high-density limit. When the momentum suppression of the nucleon degrees of freedom starts to be non-negligible due to change in the $b$-field expectation value, the HQMN result starts to deviate from the result obtained in the parity doublet model.

The HQMN model shows the chiral peak at much lower values of $\mu_{B}$. The strength of the transition is also much stronger than that obtained in the parity doublet model. The pronounced transition is the result of the suppression of the nucleon degrees of freedom through Eq. (2.5). 
Eventually at high values of baryon chemical potential the HQMN model result features a discontinuity due to the jump in the deconfinement transition. At this point the degrees of freedom change from nucleons to quarks. In the high-density limit, the model restores the proper asymptotic behavior.

While the second-order cumulant shows large sensitivity to the chiral phase transition, it is much less sensitive to the first-order deconfinement transition. This is because the transition is strictly connected to the potential $V_{b}$ and is driven by the expectation value of $b$. By construction, the potential is only symmetric with respect to a discrete symmetry. This is so because additional massless Goldstone modes would spoil the known low-energy chiral phenomenology of QCD.

In Fig. 5, we compare the third-order (left panel) and the fourth-order (right panel) cumulants of the net-baryon number density in the HQMN model. Shown are the results for three different values of $\alpha$ parameter, namely for the crossover (black dot-dashed line), second-order transition at the critical point (red dashed line), first-order transition (blue solid line). The $x$-axis is normalized by corresponding critical value of the baryon chemical potential, $\mu_{B}^{c}$. Note that while the secondorder cumulant was positive at all values of $\mu_{B}$, the higher-order cumulants can in principle both turn negative. In the case of the first-order transition, the third- and fourth-order cumulants are discontinues.

The third- and fourth-order cumulants for the second-order transition diverges at both sides of the transition point. The former one changes sign from positive to negative at the chiral transition point. In the case of the crossover, $\chi_{3}^{\mu_{B}}$ rapidly increases in the chirally broken phase, then drops and exhibits a dip at negative value in the chirally restored phase. The fourth-order cumulant exhibits similar structure, but develops a smaller secondary peak in the chirally restored phase. Note that these results are qualitatively similar to the results obtained in other chiral models incorporating the statistical confinement as, e.g., [15].

\section{Conclusions}

We have discussed the mean-field-approximation thermodynamics of the Hybrid Quark-MesonNucleon (HQMN) model at low temperature and finite baryon chemical potential. The model considers nuclear matter in the parity-doublet fashion, and quark matter in the standard quark-meson one. These are coupled together via an additional non-dynamical scalar field, whose role is to suppress quarks at low, and nucleons at high, values of temperature and baryon chemical potential.

We have studied the equation of state and the phase diagram in the HQMN model. We have shown that the chiral and deconfinement transitions are always separated. The found that the $\alpha$ parameter plays a role in changing the order of the chiral phase transit on. Namely, for low values, the transition is of first order, then at some value the model exhibits a critical point and the transition is of second order, and eventually becomes a smooth crossover at high values of the parameter. On the other hand, the deconfinement transition is always a first-order transition. We emphasize that the separation might of these two transitions might indicate the existence of the quarkyonic phase [16].

We have also studied the higher-order cumulants of the net-baryon number. We find that due to the suppression of the nucleon degrees of freedom through the momentum cutoff, the model yields much stronger chiral phase transition, when compared to the purely hadronic parity doublet 
model. The deconfinement transition, on the other hand, is found to be much less sensitive and less pronounced in the higher-order cumulants.

In this contribution, we considered an isospin-symmetric system of two flavors. In order to make the HQMN model suitable to astrophysical applications, such as neutron stars [17] or supernovae [18], it has to be extended to an arbitrary isospin asymmetry. Moreover, the appearance of heavier flavors, i.e., strangeness, must also be taken into account. Such extension within the paritydoublet model was recently formulated [19]. However, the role of strange degrees of freedom in astrophysics is still speculative, and is a matter of current investigation [20].

\section{Acknowledgments}

MM acknowledges financial support from the organizers of the CPOD 2017 conference. This work is supported by the Polish National Science Center grant DEC-2013/10/A/ST2/00106 and UMO-2014/13/B/ST9/02621. MM was also partly supported by the IFT grant no. 0420-2019-16.

\section{References}

[1] G. Aarts, C. Allton, S. Hands, B. Jäger, C. Praki and J. I. Skullerud, Phys. Rev. D 92, no. 1, 014503 (2015) doi:10.1103/PhysRevD.92.014503 [arXiv:1502.03603 [hep-lat]].

[2] G. Aarts, C. Allton, D. De Boni, S. Hands, B. Jäger, C. Praki and J. I. Skullerud, JHEP 1706, 034 (2017) doi:10.1007/JHEP06(2017)034 [arXiv:1703.09246 [hep-lat]].

[3] C. E. Detar and T. Kunihiro, Phys. Rev. D 39, 2805 (1989). doi:10.1103/PhysRevD.39.2805

[4] D. Jido, T. Hatsuda and T. Kunihiro, Phys. Rev. Lett. 84, 3252 (2000) doi:10.1103/PhysRevLett.84.3252 [hep-ph/9910375].

[5] D. Jido, M. Oka and A. Hosaka, Prog. Theor. Phys. 106, 873 (2001) doi:10.1143/PTP.106.873 [hep-ph/0110005].

[6] D. Zschiesche, L. Tolos, J. Schaffner-Bielich and R. D. Pisarski, Phys. Rev. C 75, 055202 (2007) doi:10.1103/PhysRevC.75.055202 [nucl-th/0608044].

[7] C. Sasaki and I. Mishustin, Phys. Rev. C 82, 035204 (2010) doi:10.1103/PhysRevC.82.035204 [arXiv:1005.4811 [hep-ph]].

[8] C. Sasaki, H. K. Lee, W. G. Paeng and M. Rho, Phys. Rev. D 84, 034011 (2011) doi:10.1103/PhysRevD.84.034011 [arXiv:1103.0184 [hep-ph]].

[9] S. Gallas, F. Giacosa and G. Pagliara, Nucl. Phys. A 872, 13 (2011) doi:10.1016/j.nuclphysa.2011.09.008 [arXiv:1105.5003 [hep-ph]].

[10] F. Giacosa, Prog. Part. Nucl. Phys. 67, 332 (2012) doi:10.1016/j.ppnp.2011.12.039 [arXiv:1111.4944 [hep-ph]].

[11] Y. Motohiro, Y. Kim and M. Harada, Phys. Rev. C 92, no. 2, 025201 (2015) Erratum: [Phys. Rev. C 95, no. 5, 059903 (2017)] doi:10.1103/PhysRevC.92.025201, 10.1103/PhysRevC.95.059903 [arXiv:1505.00988 [nucl-th]].

[12] J. Weyrich, N. Strodthoff and L. von Smekal, Phys. Rev. C 92, no. 1, 015214 (2015) doi:10.1103/PhysRevC.92.015214 [arXiv:1504.02697 [nucl-th]]. 
[13] S. Benic, I. Mishustin and C. Sasaki, Phys. Rev. D 91, no. 12, 125034 (2015) doi:10.1103/PhysRevD.91.125034 [arXiv:1502.05969 [hep-ph]].

[14] M. Marczenko and C. Sasaki, arXiv:1711.05521 [hep-ph].

[15] V. Skokov, B. Friman and K. Redlich, Phys. Rev. C 83, 054904 (2011) doi:10.1103/PhysRevC.83.054904 [arXiv:1008.4570 [hep-ph]].

[16] A. Andronic et al., Nucl. Phys. A 837, 65 (2010) doi:10.1016/j.nuclphysa.2010.02.005 [arXiv:0911.4806 [hep-ph]].

[17] M. A. R. Kaltenborn, N. U. F. Bastian and D. B. Blaschke, Phys. Rev. D 96, no. 5, 056024 (2017) doi:10.1103/PhysRevD.96.056024 [arXiv:1701.04400 [astro-ph.HE]].

[18] T. Fischer et al., arXiv:1711.07411 [astro-ph.HE].

[19] C. Sasaki, arXiv:1707.05081 [hep-ph].

[20] N. U. F. Bastian, D. B. Blaschke, M. Cierniak, T. Fischer, M. A. R. Kaltenborn, M. Marczenko and S. Typel, arXiv:1710.09189 [astro-ph.HE]. 